\title{
CICLOS ECONÔMICOS E MERCADO DE CRÉDITO: A ATUAÇÃO DOS BANCOS PÚBLICOS
}

\author{
ECONOMIC CYCLES AND THE CREDIT MARKET: THE STATE BANK'S OPERATION
}

\author{
José Alves Dantas ${ }^{1}$ \\ Universidade de Brasília (UnB) \\ josealvesdantas@unb.br
}

\author{
Carolina Soares Duarte \\ Universidade de Brasília (UnB) \\ Carol.soares.duarte@gmail.com
}

\author{
Bruno Vinícius Ramos Fernandes \\ Universidade de Brasília (UnB) \\ brunoramos@unb.br
}

\begin{abstract}
RESUMO
O objetivo deste estudo foi verificar se os bancos públicos atuam de forma anticíclica, comparativamente aos bancos privados, em relação à concessão de crédito e ao reconhecimento da Provisão para Créditos de Liquidação Duvidosa (PCLD). Para a realização dos testes empíricos, por meio de modelos com dados em painel, foram utilizadas informações semestrais, de 2000 a 2017, de 118 bancos. Apesar dos sinais dos coeficientes apontarem (i) relação negativa entre a variação da carteira de crédito e o nível de atividade econômica e (ii) associação positiva entre a parcela discricionária da provisão para perdas e o nível de atividade econômica, por parte dos bancos públicos, tais resultados não registraram relevância estatística, não confirmando as hipóteses de que os bancos públicos apresentariam comportamento anticíclico, comparativamente aos bancos privados. Os resultados contribuem para o avanço da literatura sobre o tema, auxiliando na compreensão do comportamento dos bancos públicos, que assumem papel relevante na indústria bancária brasileira.
\end{abstract}

Palavras-Chave: Bancos. Crédito. PCLD. Ciclos Econômicos. Ciclicidade.

\begin{abstract}
This study's purpose was to verify whether state banks act in a countercyclical manner, compared to private banks, in relation to the granting of credit and the recognition of impairment. For the empirical tests, by means of panel data models, semiannual information was used, from 2000 to 2017, of 118 banks. Although the signs of the coefficients indicate (i) a negative relation between the variation in the credit portfolio and the level of economic activity, and (ii) a positive association between the discretionary impairment for losses and the level of economic activity by state banks, these results were not statistically significant, not confirming the hypotheses that state banks would exhibit anticyclical behavior compared to private banks. The results contribute to the advancement of the literature on the subject, helping to understand the behavior of state banks, which play a relevant role in the Brazilian banking industry.
\end{abstract}

Keywords: Banks. Credit. Impairment. Business Cycles. Cyclicity.

${ }^{1}$ Prédio da Face - Campus Universitário Darcy Ribeiro - Asa Norte - Brasília (DF) - CEP 70910-900 


\section{INTRODUÇÃO}

As instituições financeiras exercem papel fundamental para que sejam alcançados os objetivos das sociedades contemporâneas, na medida em que atuam na gestão dos recursos financeiros disponíveis, gerindo-os de maneira a contribuir para a alocação ótima desses recursos na economia (CHAVES, 2014). Um sistema financeiro eficiente é uma das premissas para o desenvolvimento dos países, tendo em vista a atividade de intermediação financeira, absorvendo os recursos dos poupadores e alocando aos demandantes de capital, proporcionando o fluxo de recursos entre poupadores e investidores. Isso contribui para o lucro e o crescimento dessas entidades, mas também para o desenvolvimento da economia e da sociedade.

Dentre os assuntos que podem ser explorados acerca da atuação dos bancos, pode-se destacar a verificação das divergências entre os comportamentos dos bancos públicos e privados, tendo em vista que os bancos públicos buscam objetivos além de puro lucro, conforme argumentam Vasconcelos et al. (2004), Yeyati, Micco e Panizza (2007), Matthey (2010), Jayme Jr. e Crocco (2010), Martins, Bortoluzzo e Lazzarini (2014), entre outros.

Levando-se em consideração essa potencial divergência no comportamento entre os bancos públicos e privados, é possível levantar questionamentos acerca da atuação dessas instituições diante dos ciclos econômicos. A esse respeito, Micco e Panizza (2006) destacam que há evidências indicando que os bancos públicos podem atuar de forma anticíclica quando há retração econômica, diferentemente dos bancos privados, que, conforme Oliveira (2006), são predominantemente pró-cíclicos, ou seja, quando há um crescimento econômico, há um aumento da concessão de crédito, e em momentos de recessão, há uma contração na oferta de crédito, o que tende a intensificar ainda mais as crises financeiras. Isso também é corroborado por Beatty e Liao (2011) e Bushman e Williams (2012), ao afirmarem que em momentos de crescimento econômico os bancos tendem a conceder empréstimo com mais facilidade para seus clientes e, em momentos de crise, nos quais as pessoas tendem a demandar mais crédito, os bancos diminuem a concessão de empréstimos, o que acaba por agravar ainda mais os momentos de retração econômica.

Destaca-se ainda que é possível relacionar o volume de crédito concedido e o risco da carteira. Para Lis, Pagés e Saurina (2000), a política de crédito de um banco é o fator mais importante relacionado à oferta de crédito e, conforme Bikker e Metzemakers (2005), há uma relação positiva entre a Provisão para Créditos de Liquidação Duvidosa (PCLD) e a concessão de crédito. Para Laeven e Majnoni (2003), em um banco prudente o crescimento da PCLD deve estar relacionado positivamente à taxa de crescimento dos empréstimos, tendo em vista que o 
rápido crescimento na concessão dos empréstimos geralmente está associado à diminuição da qualidade de sua carteira e à exigência de esforços mais rígidos de monitoramento. Ademais, conforme Bushman e Williams (2012), quando o valor da PCLD estimado não é suficiente para cobrir as perdas reais em momentos de recessão econômica, o aumento no reconhecimento dessa perda induz os bancos a reduzirem o nível de empréstimo e acabam por agravar ainda mais a crise.

Nesse contexto, tendo em vista o comportamento natural do mercado de crédito em relação aos ciclos econômicos, bem como as especificidades de atuação dos bancos sob controle estatal, o presente estudo tem como objetivo avaliar se os bancos públicos brasileiros atuam de maneira anticíclica, comparativamente aos bancos privados, no que se diz respeito à concessão de crédito e ao reconhecimento da PCLD discricionária.

É esperado que os bancos públicos adotem medidas anticíclicas, pois eles buscam objetivos além de puro lucro, como ampliar as oportunidades de desenvolvimento financeiro de seus beneficiários de empréstimos e programas, podendo exercer papel anticíclico e estabilizador em momentos de dificuldades (JAYME JR.; CROCCO, 2010). A premissa para essa expectativa é que os bancos públicos não possuem apenas o objetivo de se expandir e minimizar seus riscos, esperando-se que eles desempenhem seu papel atuando de modo a favorecer a estabilidade econômica e a minimizar os impactos decorrentes de uma recessão.

Para o alcance desses objetivos, serão realizados testes empíricos, por meio de estimação de modelos com dados em painel, utilizando informações semestrais, de 2000 a 2017 , de 118 bancos em funcionamento em dezembro de 2017, tendo como base os dados disponibilizados pelo Banco Central do Brasil (BCB) no relatório IF.Data.

\section{REFERENCIAL TEÓRICO}

\subsection{Ciclos Econômicos e a Ciclicidade dos Fenômenos}

Conforme Araújo (2014), o ciclo econômico refere-se a flutuações econômicas em sentido amplo, decorrentes de variações na produção ou na atividade econômica ocorridas durante determinado período. Essas flutuações envolvem movimentos entre períodos de crescimento econômico relativamente rápido e períodos de relativa estagnação ou recessão, variações essas que ocorrem em torno de uma tendência de crescimento de longo prazo. Ainda conforme o autor, essas flutuações são geralmente mensuradas pelo Produto Interno Bruto (PIB).

Com relação ao comportamento de determinadas variáveis frente aos ciclos econômicos, 
ressalta-se os fenômenos da próciclicidade e anticiclicidade. Aquela consiste no comovimento positivo entre uma determinada variável e a atividade econômica, fenômeno que consiste no excesso de tendências cíclicas na atividade econômica agregada, intensificando as flutuações dos ciclos econômicos (BEBCZUK; ET AL., 2011; BUSHMAN; WILLIAM, 2012). Longbrake e Rossi (2011) também tratam sobre o assunto, afirmando que políticas e mecanismos de governança são pró-cíclicos na medida em que intensificam os desequilíbrios observados em momentos de expansão da economia e quando reforçam o declínio econômico em momentos de retração. Já a anticiclicidade ocorre quando estes fenômenos atuam de modo a reduzir o acúmulo nos desequilíbrios, diminuindo, assim, a amplitude dos ciclos econômicos.

\subsection{Diferenças entre os Bancos Públicos e Privados em Relação à Política de Crédito}

A análise da maneira como os bancos públicos e privados desempenham seu papel na sociedade é um assunto abordado por diversos autores, ressaltando que os bancos públicos buscam objetivos além de puro lucro. Vasconcelos et al. (2004) e Matthey (2010) discorrem que o governo pode criar bancos públicos para prestar assistência a áreas mais remotas ou a consumidores mais arriscados, atuando, assim, em áreas de menor interesse do setor privado. Ademais, segundo Yeyati, Micco e Panizza (2007), os emprestadores privados possuem incentivos limitados para financiar projetos que produzam externalidades.

Segundo Seabra, Giglio, Fernandes e Meurer (2009), existem diferenças significativas de comportamento entre os bancos públicos e privados, as quais se refletem na transmissão do crédito, interferindo, assim, nos efeitos esperados pelas ações de política monetária. Ademais, os autores concluíram que a existência das instituições financeiras públicas é um instrumento adicional que contribui para a condução da política econômica, e os benefícios sociais decorrentes do acesso ao crédito não fornecido pelo setor privado permitem manter a influência sobre a estrutura do sistema financeiro brasileiro. Jayme Jr. e Crocco (2010) destacam ainda que os bancos públicos com atividade comercial ampliam as oportunidades de desenvolvimento financeiro de seus beneficiários de empréstimos e programas e podem também exercer papel anticíclico e estabilizador em momentos de dificuldades.

No que se concerne à atuação dos bancos públicos e privados e à concessão de crédito dessas instituições, Micco e Panizza (2006) e Oliveira (2006) afirmam que há evidências de que os bancos públicos podem atuar de maneira anticíclica quando há retração econômica, diferentemente dos bancos privados. Ou seja, em períodos de expansão econômica há aumento da concessão de crédito, e, em momentos de retração, há contração na oferta de crédito, de 
modo a intensificar ainda mais as crises econômicas.

Considerando esse contexto de diferenciação entre os papéis desenvolvidos pelos bancos públicos e privados, notadamente em relação ao propósito de fomento ao desenvolvimento econômico assumido pelos primeiros, indo além da maximização dos lucros, é formulada a seguinte hipótese de pesquisa, a ser testada empiricamente:

H1: Os bancos públicos brasileiros atuam de maneira anticíclica, comparativamente aos bancos privados, no que diz respeito à concessão de crédito, registrando relação negativa entre a variação da carteira e os ciclos econômicos.

\subsection{Risco de Crédito e o Reconhecimento de Perdas em Operações de Crédito em Bancos}

O risco de crédito é definido como a possibilidade de um tomador de um empréstimo ou contraparte não cumprir suas obrigações conforme os termos acordados (BCBS, 2000). Tendo em vista que a concessão de empréstimos constitui a atividade básica dos bancos (STOLF, 2008), o risco de crédito deve ser objeto de gerenciamento cuidadoso, de modo a buscar meios para minimizá-lo de forma mais eficiente possível. Por isso, o reconhecimento da PCLD exerce um papel fundamental para que se tenha uma representação mais fidedigna da situação econômica das entidades, na medida em que ajusta o valor dos recebíveis e do resultado de acordo com a expectativa, baseada em fatores objetivos e subjetivos, do que realmente se espera receber.

Destaca-se, ainda, que as reservas constituídas para perdas em empréstimos serão utilizadas quando essas perdas vierem de fato a ocorrer. Ademais, ressalta-se que essas provisões estão diretamente relacionadas à qualidade da carteira de empréstimos (BIKKER; METZEMAKERS, 2005). Segundo Bouvatier e Lepetit (2008), as regras de provisão podem ser de duas naturezas: Backward-looking, quando baseadas nas perdas incorridas das operações e forward-looking, quando constituídas com base nas perdas esperadas. Conforme Araújo (2014), no modelo de perda incorrida são os eventos passados que determinam qual o valor da provisão a ser constituída. Já o modelo de perda esperada é mais subjetivo e admite o reconhecimento de provisões com base em expectativas futuras, independentemente de existir evidência objetiva de perda.

No Brasil, o modelo de constituição da PCLD adotado pelas instituições financeiras tem como base a Resolução CMN no 2.682/99 e pode ser considerado um modelo misto, tendo em vista que considera no momento da concessão do crédito uma provisão para perda - a depender 
do rating da operação e do tomador -, além da realização revisões periódicas para verificar o nível de risco das operações em andamento, com base no comportamento de crédito do cliente (CANECA; LUSTOSA, 2017). A norma dispõe que as instituições devem classificar as operações de crédito em ordem crescente de risco e que a PCLD deve ser constituída mensalmente, aplicando, no mínimo, os percentuais descritos na Tabela 1.

Tabela 1: Classificação das operações por nível de risco

\begin{tabular}{cccc}
\hline & & \multicolumn{2}{c}{ Dias de atraso } \\
\cline { 3 - 4 } $\begin{array}{c}\text { Nível de } \\
\text { Risco }\end{array}$ & $\begin{array}{c}\text { Provisão } \\
\text { mínima }\end{array}$ & $\begin{array}{c}\text { Prazo Residual } \\
\leq 36 \text { meses }\end{array}$ & $\begin{array}{c}\text { Prazo Residual } \\
>36 \text { meses }\end{array}$ \\
\hline AA & $0,0 \%$ & & \\
A & $0,5 \%$ & Entre 15 e 30 & Entre 30 e 60 dias \\
B & $1,0 \%$ & Entre 31 e 60 dias & Entre 61 e 120 dias \\
C & $3,0 \%$ & Entre 61 e 90 dias & Entre 121 e 180 dias \\
D & $10,0 \%$ & Entre 91 e 120 dias & Entre 181 e 240 dias \\
E & $30,0 \%$ & Entre 121 e 150 dias & Entre 241 e 300 dias \\
F & $50,0 \%$ & Entre 151 e 180 dias & Entre 301 e 360 dias \\
G & $70,0 \%$ & Superior a 180 dias & Superior a 360 dias \\
H & $100,0 \%$ & & \\
\hline
\end{tabular}

A Resolução estabelece que na classificação dos níveis de riscos devem ser considerados aspectos como a situação econômico-financeira, o grau de endividamento, a capacidade de geração de resultados, o fluxo de caixa, a administração e qualidade de controles, a pontualidade e atrasos nos pagamentos, as contingências, o setor de atividade econômica, a renda e o patrimônio (no caso de pessoa física) e o limite de crédito, relativamente ao devedor e a seus garantidores. No que se refere à operação de crédito, devem ser considerados a natureza e finalidade da transação, as características das garantias, particularmente quanto à suficiência e liquidez e o seu valor.

É importante ressaltar que as provisões mínimas da Tabela 1 são uma espécie de piso. A Resolução $\mathrm{CMN} \mathrm{n}^{\circ}$ 2.682/99 dispõe que as instituições devem constituir a provisão de acordo com os percentuais mínimos para cada nível de risco, "sem prejuízo da responsabilidade dos administradores das instituições pela constituição de provisão em montantes suficientes para fazer face a perdas prováveis na realização dos créditos". Isso incorpora ao modelo de perdas uma parcela discricionária, sob responsabilidade da administração, de forma que o valor reflita a efetiva expectativa de perdas com as operações, independentemente dos limites regulamentares.

Segundo Bushman e Williams (2012), a discricionariedade da PCLD possui dois lados. Ao mesmo tempo que viabiliza mitigar a pró-ciclicidade e facilitar a incorporação de mais 
informações sobre as perdas futuras, aumenta a probabilidade de os administradores dos bancos se comportarem de maneira oportunista, o que pode tanto prejudicar a transparência dessas instituições como trazer consequências negativas para a admissão dos riscos.

A esse respeito, Bouvatier e Lepetit (2012) verificaram que o efeito do ciclo econômico sobre as provisões é compensado pela formação de um colchão para cobrir perdas com empréstimos, apesar de poder levar a uma alta flutuação do capital dos bancos. Na mesma linha, Caneca e Lustosa (2017) afirmam que as despesas com perdas em operações de crédito dos bancos brasileiros estimulam o comportamento pró-cíclico dos empréstimos, tendo em vista que constituem menos PCLD em época de expansão econômica e provisionam mais em momentos de recessão. Isso é corroborado por Araújo (2014) que, ao testar a hipótese de pesquisa que visava verificar se o modelo para constituição da PCLD adotado no Brasil apresentava relação positiva com os ciclos econômicos, não teve sua hipótese confirmada e verificou que, na verdade, há um comportamento pró-cíclico dos bancos comerciais brasileiros.

Autores como Bouvatier e Lepetit (2012) também analisaram questões relacionadas a esse assunto e verificaram que o efeito do ciclo econômico sobre as provisões é compensado pela formação de um colchão para cobrir perdas com empréstimos, apesar de poder levar a uma alta flutuação do capital dos bancos. Além disso, Caneca e Lustosa (2017) dispõem que o Banco Central do Brasil (BCB), quando questionado acerca do comportamento do Brasil frente à crise de 2008, em que o país optou por expandir o crédito e o consumo, afirmou que o modelo de reconhecimento de perdas, instituído pela Resolução $\mathrm{CMN} \mathrm{n}^{\circ} 2.682 / 99$, permite que as instituições financeiras criem um colchão para absorver os efeitos de uma crise financeira. Os achados de Primo (2003), ao constatar que as instituições bancárias se baseiam na expectativa de resultado do exercício ao constituírem a PCLD, formando um colchão nos exercícios que têm maior resultado, o qual é utilizado em períodos com lucros menores ou com prejuízos, reforçam essa ideia.

\subsection{Diferenças entre os Bancos Públicos e Privados em Relação à PCLD Discricionária}

Araújo (2014), ao testar a hipótese de que o modelo brasileiro para constituição da PCLD apresentava relação positiva com os ciclos econômicos, não confirmou suas expectativas e constatou que, na verdade, há comportamento pró-cíclico dos bancos comerciais. Não obstante, ao verificar a relação entre o estoque de PCLD dos bancos públicos e a variação do PIB, apesar de apresentar sinal negativo, não encontrou associação estatisticamente relevante, diferentemente dos bancos privados, em que a associação negativa se mostrou estatisticamente 
relevante, o que é um indício de que pode haver divergência entre o comportamento dos bancos públicos e privados em relação à constituição da PCLD.

Este estudo se diferencia de Araújo (2014), por verificar a relação da PCLD discricionária - ao invés do montante total da PCLD - com o PIB. Isso ocorre porque, como ele visa verificar o comportamento dos bancos públicos, levando em consideração que sua atuação vai além da maximização de lucros, conforme afirmado por Jayme Jr. e Crocco (2010), a utilização desta variável faz-se mais eficiente. O argumento é que como no montante total da PCLD é considerada também a PCLD regulamentar, que representa grande parte da provisão constituída nos bancos, utilizá-la como variável acaba por avaliar mais o comportamento do modelo de provisão para perdas do que a forma como os bancos administram sua PCLD. Assim, utilizar a parcela discricionária dessa provisão - no caso específico, sua relação com os ciclos econômicos - traduz mais claramente a avaliação da influência das decisões dos gestores dessas instituições.

Nesse contexto, dadas as diferenças de atuação e propósitos entre os bancos públicos e privados, incluindo os seus efeitos no reconhecimento das perdas em operações de crédito, é formulada a segunda hipótese de pesquisa, a ser testada empiricamente:

H2: Os bancos públicos brasileiros atuam de maneira anticíclica, comparativamente aos bancos privados, no que diz respeito ao reconhecimento da PCLD, registrando relação positiva entre as despesas discricionárias com perdas de crédito e os ciclos econômicos.

\section{PROCEDIMENTOS METODOLÓGICOS}

Considerando o propósito de avaliar se os bancos públicos brasileiros atuam de maneira anticíclica, comparativamente aos bancos privados, no que se refere à concessão de crédito e ao reconhecimento da PCLD discricionária, foram desenvolvidos os modelos (3.1) e (3.4) para testar empiricamente as hipóteses de pesquisas $\boldsymbol{H}_{1}$ e $\boldsymbol{H}_{2}$, respectivamente.

\subsection{Modelo para Testar a Hipótese de Pesquisa $H_{1}$}

A primeira hipótese de pesquisa foi formulada com o objetivo de verificar se os bancos públicos brasileiros atuam de maneira anticíclica, comparativamente aos bancos privados, no que concerne à concessão de crédito. Para testar $\boldsymbol{H}_{1}$, será considerada a estimação do modelo

$$
\Delta O_{p} C_{i t}=\beta_{0}+\beta_{1} O p C_{i t}+\beta_{2} \operatorname{Prov}_{i t}+\beta_{3} S E L I C_{t}+\beta_{4} P I B_{t}+\beta_{5} P U B_{i}+\beta_{6}\left(P I B_{t} * P U B_{i}\right)+\varepsilon_{i t}
$$


Onde:

$\triangle O p C_{i t}$ : Variação proporcional da carteira de crédito, do banco $i$, no momento $t$ em relação a $t$ - 1 , representando a taxa de crescimento (ou involução) da carteira de crédito em relação ao período anterior, mensurada da seguinte forma: $\left(\left(O p C_{t}-O p C_{t-1}\right) / O p C_{t-1}\right)$.

$O p C_{i t}$ : Logaritmo natural do total das operações de crédito, do banco $i$, no momento $t$.

Prov $_{i t}$ : Razão entre o estoque da PCLD e o total das operações de crédito, do banco $i$, no período $t$.

$S E L I C_{t}$ : Taxa de juros equivalente à taxa referencial do Sistema Especial de Liquidação e de Custódia acumulada do semestre, no período $t$.

$P I B_{t}$ : Variação (evolução ou retração) do Produto Interno Bruto do país, no semestre $t$ - a medida captura, portanto, movimentos de crescimento ou de redução da riqueza produzida no país, em determinado período.

$P U B_{i}$ : Variável dummy, assumindo 0 para bancos privados e 1 para bancos públicos.

A hipótese de pesquisa $\boldsymbol{H}_{\boldsymbol{I}}$ será confirmada caso a variável de interação $\boldsymbol{P I B} * \boldsymbol{P U B}$ apresente relação negativa e estatisticamente relevante com a variável dependente $(\boldsymbol{\Delta O p C})$, demonstrando que os bancos públicos atuam de maneira anticíclica, na medida em que registram relação inversa entre a variação da carteira e os ciclos econômicos, representados pela variação do PIB. O uso da variável $P I B$, ao invés de uma dummy representativa de ciclos de expansão ou retração econômica se justifica pelo fato de o propósito do estudo ser identificar se há comportamento anticíclico por parte dos bancos públicos, em comparação aos bancos privados. A hipótese $H_{1}$ considera que em períodos de maior expansão econômica os bancos privados registram maior crescimento de sua carteira de crédito do que os bancos públicos, e que em períodos de maior retração econômica ocorre o inverso.

Com relação às variáveis independentes de controle que compõem o modelo (3.1), o total das operações de crédito $(\boldsymbol{O P C})$ justifica-se pela expectativa de que o tamanho da carteira de crédito de uma instituição influenciar na variação dessas operações, na medida em que é esperado que bancos maiores possuam menor variação proporcional de suas carteiras do que bancos menores.

Já para o nível de provisão (Prov) é esperada relação positiva com a variação da carteira de crédito, seguindo Lis, Pagés e Saurina (2000) e Bikker e Metzemakers (2004), para quem a política de crédito de um banco é o fator mais importante relacionado à oferta de crédito, sendo possível afirmar que há associação entre a PCLD e o aumento da concessão de crédito. Ademais, para Laeven e Majnoni (2003), em um banco prudente o crescimento da PCLD deve estar relacionado positivamente à taxa de crescimento dos empréstimos, tendo em vista que o rápido crescimento na concessão dos empréstimos geralmente está associado à diminuição da 
qualidade de sua carteira e à exigência de esforços mais rígidos de monitoramento.

A variável $\boldsymbol{P I B}$ é utilizada para representar os ciclos econômicos, tendo sido utilizada com essa finalidade por Bouvatier e Lepetit (2012), Bushman e Williams (2012) e Araújo (2014). Ademais, o crescimento do PIB pode ser considerado o indicador mais útil para representar o ciclo econômico (BIKKER; HU, 2002). A taxa SELIC também pode ser uma proxy representativa da situação econômica do país pois, conforme Mendonça et al. (2005), a definição dessa taxa se dá na medida em que seja capaz de influenciar o comportamento do nível de preços e a atividade econômica, além de servir como base para a definição de todas as outras taxas da economia.

\subsection{Modelo para Testar a Hipótese de Pesquisa $\mathrm{H}_{2}$}

Para testar $\boldsymbol{H}_{2}$, que procura avaliar se os bancos públicos atuam de maneira anticíclica, comparativamente aos bancos privados, em relação ao reconhecimento da PCLD, o primeiro passo consiste na mensuração da PCLD discricionária - determinada pela aplicação dos percentuais definidos na Resolução CMN n ${ }^{\circ}$ 2.682/99 sobre o estoque total da PCLD para, assim, obter-se o valor da PCLD regulamentar. Como o montante da PCLD é composto pela parcela obrigatória e pela parcela discricionária, obtém-se a parcela discricionária subtraindo a parcela regulamentar apurada do total da PCLD registrada pelos bancos, conforme equações (3.2) e (3.3):

$$
\begin{aligned}
& P C L D_{i t}=P C L D d_{i t}+P C L D n d_{i t} \\
& P C L D d_{i t}=P C L D_{i t}-P C L D n d_{i t}
\end{aligned}
$$

Onde:

PCLD $i t$ : Total da PCLD contabilizada no banco $i$, no momento $t$.

$P C L D d_{i t}$ : Parcela da PCLD discricionária, do banco $i$, no momento $t$.

PCLDnd $d_{i t}$ : valor da PCLD não discricionária, do banco $i$, no momento $t$, obtido por meio da aplicação do percentual regulamentar instituído na Resolução CMN nº 2682/99, conforme Tabela 1, aplicado sobre os montantes das contas dos níveis de risco da instituição.

Identificada a parcela discricionária, é desenvolvido o modelo (3.4) para testar $\boldsymbol{H}_{2}$ :

$$
\begin{aligned}
\text { PCLDd }_{i t}= & \beta_{0}+\beta_{1} O p C_{i t}+\beta_{2} \Delta O p C_{i t}+\beta_{3} \text { Prov }_{i t}+\beta_{4} \text { Inad }_{i t}+\beta_{5} \text { SELIC }_{t} \\
& +\beta_{6} P_{I} B_{t}+\beta_{7} P U B_{i}+\beta_{8}\left(\text { PIB }_{t} * P U B_{i}\right)+\varepsilon
\end{aligned}
$$

Onde: 
$P C L D d_{i t}$ : Nível da provisão de crédito para liquidação duvidosa discricionária, obtida por meio da razão entre a

PCLD discricionária, apurada conforme equação (3.3), e o total das operações de crédito do período, do banco $i$, no período $t$.

$O p C_{i t}$ : Logaritmo natural do total das operações de crédito, do banco $i$, no momento $t$.

$\Delta O p C_{i t}$ : Variação proporcional da carteira de crédito, do banco $i$, no momento $t$ em relação a $t$ - 1 .

Prov $_{i t}$ : Razão entre o estoque da PCLD e o total das operações de crédito, do banco $i$, no período $t$.

$\operatorname{Inad}_{i t}$ : Razão entre o total das operações classificadas entre os níveis de risco "E" e " $\mathrm{H}$ " e o total das operações de crédito, do banco $i$, no período $t$.

$S E L I C_{t}$ : Taxa de juros equivalente à taxa referencial do Sistema Especial de Liquidação e de Custódia acumulada do semestre, no período $t$.

$P I B_{t}$ : Variação (evolução ou retração) do Produto Interno Bruto do país, no semestre $t$ - a medida captura, portanto, movimentos de crescimento ou de redução da riqueza produzida no país, em determinado período.

$P U B_{i}$ : Variável dummy, assumindo 0 para bancos privados e 1 para bancos públicos.

Para concluir sobre $\boldsymbol{H}_{2}$, a variável de interesse é representada pela interação $\boldsymbol{P I B} * \boldsymbol{P U B}$. Caso se constate relação positiva e estatisticamente relevante com a PCLD discricionária $(\boldsymbol{P C L D d})$, se confirmará que os bancos públicos, comparativamente aos bancos privados, promovem, em períodos de expansão econômica, o aumento PCLD discricionária, e, em momentos de retração, a diminuição dessa provisão. Isso indicaria a ocorrência do afirmado por Bouvatier e Lepetit (2012), que constataram o efeito do ciclo econômico sobre as provisões, compensado pela formação de um colchão para cobrir perdas com empréstimos. Conforme Araújo (2014), como é permitido aos gestores dos bancos relativa discricionariedade na constituição da PCLD, essa acaba por ser influenciada por uma série de fatores, resultando na constituição desses colchões a serem utilizadas em momentos de pior comportamento da economia.

No tocante às variáveis de controle, é esperada relação positiva entre a provisão discricionária $(\boldsymbol{P C L D d})$ e as variáveis representativas da variação da carteira de crédito $(\boldsymbol{\Delta O P C})$ e o volume das operações de crédito $(\boldsymbol{O p} \boldsymbol{C})$, justificadas pela natural associação entre o tamanho da carteira e sua evolução com o volume de provisões reconhecidas, inclusive a parcela discricionária.

O nível de provisão (Prov) é apresentado como variável independente, na medida em que a variável considera o total da PCLD e, como grande parte dessa provisão é constituída pela provisão regulamentar, instituída pela Resolução CMN n ${ }^{\circ}$ 2.682/99, um alto nível de provisão pode indicar que há risco um considerável das operações que compõem a carteira de crédito, o que pode levar os gestores a optarem por constituir uma maior provisão discricionária, e vice- 
versa.

A inadimplência (Inad) também pode exercer influência no comportamento da variável dependente, considerando que um grande volume de recebíveis classificados em níveis de risco caracterizados como atraso superiores a 90 dias (no caso, nos níveis "E" a "H") pode representar a má qualidade da carteira de crédito da instituição, funcionando como um indicativo de maior risco para a instituição e levando a administração a aumentar sua PCLD discricionária.

Ademais, conforme já destacado, a variação do PIB pode ser considerada como o indicador mais útil para representar o ciclo econômico (BIKKER; HU, 2002), sendo esperado que em momentos de maior crescimento econômico o nível de provisão discricionário seja mais relevante. Para a variável SELIC, também entendida como uma proxy representativa da situação econômica do país, conforme Mendonça et al. (2005), é esperada uma associação positiva com a PCLD discricionária, pela mesma razão destacada em relação à variação do PIB, tendo em vista a natural associação entre essas variáveis macroeconômicas.

\subsection{Amostra}

Para a estimação desses modelos, são utilizados dados de 118 bancos brasileiros em atividade na data-base dezembro de 2017, incluindo bancos comerciais, bancos múltiplos, caixas econômicas e bancos de desenvolvimento. Foram utilizadas informações semestrais de 2000 a 2017, disponíveis no relatório IF.Data, na página do Banco Central do Brasil (BCB) na internet.

\section{ANÁLISE DOS RESULTADOS}

\subsection{Estatísticas Descritivas}

A primeira etapa dos testes consiste em apurar as variáveis dependentes e independentes que integram os modelos (3.1) e (3.4), tendo por base a amostra apresentada na Seção 3.3. As estatísticas descritivas são consolidadas na Tabela 2.

Tabela 2: Estatísticas descritivas das variáveis dos modelos (3.1) e (3.4)

\begin{tabular}{|c|c|c|c|c|c|c|c|c|c|}
\hline $\begin{array}{l}\text { Descrição } \\
\text { Variáveis }\end{array}$ & $O p C_{i t}$ & $\Delta O p C_{i t}$ & Provit $_{1}$ & PCLDd $_{i t}$ & Inadit $_{\text {t }}$ & $S E L I C_{t}$ & $P U B_{t}$ & $\boldsymbol{P I B}_{t}$ & $P^{\prime} B_{t} * P U B_{i}$ \\
\hline Média & 20,3738 & 0,1148 & 0,0575 & 0,0026 & 0,0634 & 0,0643 & 0,1420 & 0,0223 & 0,0034 \\
\hline Mediana & 20,4400 & 0,0652 & 0,0382 & 0,0000 & 0,0383 & 0,0594 & 0,0000 & 0,0230 & 0,0000 \\
\hline Máximo & 27,2800 & 3,1161 & 0,7119 & 0,2409 & 0,8291 & 0,1182 & 1,0000 & 0,0852 & 0,0852 \\
\hline Mínimo & 10,9800 & $-0,7072$ & 0,0000 & $-0,1230$ & 0,0000 & 0,0351 & 0,0000 & $-0,0558$ & $-0,0558$ \\
\hline $\begin{array}{l}\text { Desvio } \\
\text { Padrão }\end{array}$ & 2,5722 & 0,3612 & 0,0739 & 0,0102 & 0,0914 & 0,0191 & 0,3491 & 0,0335 & 0,0149 \\
\hline
\end{tabular}


Onde: $\boldsymbol{O} \boldsymbol{p} \boldsymbol{C}_{i t}$ corresponde ao logaritmo natural do total das operações de crédito, do banco $i$, no momento $t ; \boldsymbol{\Delta} \boldsymbol{O p} \boldsymbol{C}_{i t}$ : é a variação proporcional da carteira de crédito, do banco $i$, no momento $t$ em relação a $t$ - 1 ; Provit representa a razão entre o estoque da PCLD do período e o total das operações de crédito, do banco $i$, no momento $t$; $\boldsymbol{P C L D d _ { i t }}$ é o nível da provisão de crédito para liquidação duvidosa discricionária, obtida por meio da razão entre a PCLD discricionária e o total das operações de crédito do período, do banco $i$, no período $t$; Inadit é a razão entre o total das operações classificadas entre os níveis de risco "E" e "H" e o total das operações de crédito, do banco $i$, no período $t ; \boldsymbol{S E L I C} \boldsymbol{C}_{t}$ corresponde à taxa básica de juros da economia acumulada no semestre $t ; \boldsymbol{P} \boldsymbol{U} \boldsymbol{B}_{i}$ é uma variável dummy, assumindo 0 para bancos privados e 1 para bancos públicos; e $\boldsymbol{P I B} t$ corresponde à variação do Produto Interno Bruto semestral do país, no período $t$.

As estatísticas descritivas revelam que entre 2000 e 2017 houve um aumento médio de $11 \%$ ao semestre (a.s.) no saldo das operações de crédito $(\boldsymbol{A O P C})$. Excluindo os efeitos dos valores extremos, a mediana demonstra que o crescimento médio da carteira de crédito dos bancos brasileiros foi de 6,5\% a.s. Em relação ao nível de provisão (Prov) da carteira de crédito, a mediana evidencia que os bancos reconhecem como perdas cerca de 3,8\% do estoque. Já a parcela discricionária da provisão (PCLDd) registra um percentual médio de $0,26 \%$, sendo que menos da metade das instituições reconhecem provisão para perdas acima do mínimo regulamentar - critério utilizado como referência para definir a discricionariedade.

A variável Inad, obtido pela razão entre o total das operações classificadas entre os níveis de risco "E" e "H" e o total das operações de crédito, indica que as operações classificadas nesses níveis de risco representaram, em média, 6,3\% do total das operações, com mediana de 3,8\%. Percebe-se a compatibilidade nos resultados obtidos, considerando que o nível de provisão (Prov) e a inadimplência (Inad) apresentaram valores bastante semelhantes.

Destaca-se, por fim, que os bancos públicos, federais e estaduais, representam 14\% do total das observações consideradas no estudo.

\subsection{Estimação do Modelo (3.1) e Teste da Hipótese $H_{1}$}

Para testar a hipótese $\boldsymbol{H}_{\boldsymbol{l}}$, no sentido de verificar se os bancos públicos brasileiros, comparativamente aos bancos privados, atuam de maneira anticíclica no que se refere à concessão de crédito, registrando relação inversa entre a variação da carteira e os ciclos econômicos, é estimado o modelo (3.1), utilizando dados em painel, nos métodos pooled e efeitos fixos seccionais, cujos resultados são consolidados na Tabela 3. Para esse fim, considerando as evidências de autocorrelação nos resíduos, foi utilizado o método SUR PCSE, que gera parâmetros robustos, mesmo na presença de autocorrelação e heterocedasticidade nos resíduos. 
Tabela 3: Resultados da Estimação do modelo (3.1)

\begin{tabular}{|c|c|c|}
\hline$\Delta O p C_{i, t}=\beta_{0}+$ & $\Delta O p C_{i, t}=\beta_{0}+\beta_{1} O p C_{i t}+\beta_{2} \operatorname{Prov}_{i t}+\beta_{3} S E L I C_{t}+\beta_{4} P I B_{t}+\beta_{5} P U B_{i}+\beta_{6}\left(P I B_{t} * P U B_{i}\right)+\varepsilon_{i t}$ & $\left.U B_{i}\right)+\varepsilon_{i t}$ \\
\hline & Pooled & Efeitos Fixos \\
\hline$C$ & $\begin{array}{c}0,2678 \\
(0,0028) \\
* * *\end{array}$ & $\begin{array}{c}0,3001 \\
(0,0001) \\
* * *\end{array}$ \\
\hline$O p C_{i t}$ & $\begin{array}{c}-0,0078 \\
(0,0348) \\
* *\end{array}$ & $\begin{array}{c}-0,0069 \\
(0,0647) \\
*\end{array}$ \\
\hline Prov $_{i t}$ & $\begin{array}{l}-0,7550 \\
(0,0000) \\
* * *\end{array}$ & $\begin{array}{c}-0,7189 \\
(0,0000) \\
* * *\end{array}$ \\
\hline$S E L I C_{t}$ & $\begin{array}{c}0,4124 \\
(0,3921)\end{array}$ & \\
\hline$P I B_{t}$ & $\begin{array}{c}1,1340 \\
(0,0004) \\
* * * \\
\end{array}$ & \\
\hline$P U B_{i}$ & $\begin{array}{c}-0,0122 \\
(0,4470)\end{array}$ & $\begin{array}{c}-0,0170 \\
(0,3261)\end{array}$ \\
\hline$P I B_{t} * P U B_{i}$ & $\begin{array}{c}-0,4134 \\
(0,2513)\end{array}$ & $\begin{array}{l}-0,3739 \\
(0,3374)\end{array}$ \\
\hline $\mathrm{N}^{\circ}$ de Entidades & 118 & 118 \\
\hline $\mathrm{N}^{\mathrm{o}}$ de Observações & 3.236 & 3.236 \\
\hline Período & $2000 / 2017$ & $2000 / 2017$ \\
\hline $\mathrm{R}^{2}$ & 0,0415 & 0,0640 \\
\hline $\mathrm{R}^{2}$ Ajustado & 0,0398 & 0,0529 \\
\hline Estatística-F & 23,3235 & 5,7554 \\
\hline F (p-valor) & 0,0000 & 0,0000 \\
\hline
\end{tabular}

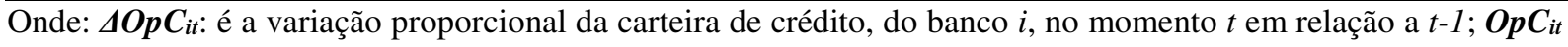
corresponde ao logaritmo natural do total das operações de crédito, do banco $i$, no momento $t$; Provit representa a razão entre o estoque da PCLD do período e o total das operações de crédito, do banco $i$, no momento $t ; \boldsymbol{S E L I C}_{t}$ corresponde à taxa básica de juros da economia acumulada no semestre $t ; \boldsymbol{P I B}_{t}$ corresponde à variação do Produto Interno Bruto semestral do país, no período $t$; e $\boldsymbol{P} \boldsymbol{U} \boldsymbol{B}_{i}$ é uma variável $d u m m y$, assumindo 0 para bancos privados e 1 para bancos públicos. Nível de significância: $* * * 1 \%, * * 5 \%, * 10 \%$. P-valores entre parênteses.

Com base nos resultados expostos na Tabela 3, verificou-se que a variável de interação $\boldsymbol{P I B} * \boldsymbol{P U B}$, apesar de possuir sinal negativo, não apresentou relação estatisticamente relevante com a variável dependente $(\boldsymbol{\Delta O P C})$, que representa a variação do total das operações de crédito. Por isso, não foi possível comprovar que os bancos públicos atuam de maneira anticíclica, comparativamente aos bancos privados, no que diz respeito à concessão de crédito e, deste modo, $\boldsymbol{H}_{1}$ não foi corroborada. De se ressaltar que os testes realizados avaliam a relação entre a variação do estoque de crédito e o nível de atividade econômica, em termos contemporâneos, o que representa uma espécie de limitação, tendo em vista que os potenciais efeitos do comportamento do PIB na política de crédito dos bancos públicos pode se dar em momento não exatamente contemporâneo - pode também procurar se antecipar aos ciclos econômicos ou responder posteriormente aos seus efeitos.

Em relação à variável independente representativa da carteira de crédito $(\boldsymbol{O p C})$, constatou-se uma relação negativa com a variável dependente $(\Delta O p C)$. Isso indica que quanto 
maior a carteira de crédito, menor sua variação, e vice-versa, o que é razoável, tendo em vista que bancos com carteira de crédito mais relevante tendem a ter menos oscilações proporcionais em seu estoque de operações. Os resultados obtidos registraram relevância estatística, tanto na estimação com efeitos fixos nos períodos e no pooled.

Já o nível de provisão (Prov), representado pela razão entre o estoque de PCLD e o total das operações de crédito, possui relação negativa e estatisticamente relevante com a variação total das operações de crédito, ou seja, quanto maior o nível de provisão, menor a variação dessas operações, o que não corrobora a afirmação de Bikker e Metzemakers (2005) no sentido de haver uma relação positiva entre a PCLD e o aumento da concessão de crédito. Esses resultados sugerem que quanto maior o risco da carteira, menor sua taxa de crescimento.

A variável independente representativa da taxa básica de juros da economia (SELIC), no modelo pooled, não apresentou relação estatisticamente relevante com a variável dependente. Com relação ao nível de atividade econômica (PIB), considerando todos os bancos da amostra, verificou-se uma associação positiva e estatisticamente relevante entre a variável dependente $(\boldsymbol{\Delta O} \boldsymbol{C})$, indicando que em momentos de maior expansão econômica os bancos aumentam o volume de concessão de crédito e em momentos de retração econômica restringem sua política de crédito. Esses achados corroboram as afirmações de Beatty e Liao (2011) e Bushman e Williams (2012) no sentido de que, em momentos de crescimento econômico, os bancos tendem a conceder empréstimo com mais facilidade para seus clientes e, em momentos de crise, nos quais as pessoas tendem a demandar mais por crédito, os bancos diminuem a concessão de empréstimos. A hipótese testada neste estudo, em relação aos bancos públicos, é exatamente o inverso previsto nessa premissa, ou seja, avalia se essas instituições atuam de forma contrária ao esperado - de forma anticíclica.

Por meio dos resultados obtidos da relação entre a variável $\boldsymbol{P} \boldsymbol{U} \boldsymbol{B}$ e a variável dependente, percebe-se que não é possível afirmar que a carteira de crédito dos bancos públicos apresenta maior variação do que a dos bancos privados, tendo em vista que a relação entre a variável dummy e a variável dependente não apresentou relevância estatística.

Ademais, destaca-se que o fato de a relação entre a variação do PIB e a variação total das operações de crédito ser positiva e estatisticamente relevante e a relação entre a variação da carteira de crédito e a variável de interação $\boldsymbol{P I B} * \boldsymbol{P U} \boldsymbol{B}$ não possuir relevância estatística, pode ser um indicativo de que os bancos públicos atuam de forma anticíclica. Isso se dá, pois, como se teve uma relação positiva entre a variável $\boldsymbol{P I B}$ e a variável $\boldsymbol{\Delta O} \boldsymbol{P C}$, era razoável que, se os bancos públicos também se comportassem dessa forma, eles também apresentariam relação 
positiva, e o fato de a associação não ter significância estatística é um indicativo de que pode haver diferença entre o comportamento dos bancos públicos e privados, embora não seja possível afirmar que há, de fato, a atuação anticíclica dos bancos públicos brasileiros.

\subsection{Estimação do Modelo (3.4) e Teste da Hipótese $\mathrm{H}_{2}$}

Levando em consideração que $\boldsymbol{H}_{2}$ visa analisar se os bancos públicos brasileiros atuam de maneira anticíclica, comparativamente aos bancos privados, no que concerne ao reconhecimento da PCLD, foi estimado o modelo (3.4) com o método SUR(PCSE), dadas as evidências de autocorrelação nos resíduos. Os resultados obtidos estão consolidados na Tabela 4.

Tabela 4: Resultados da Estimação do modelo (3.4)

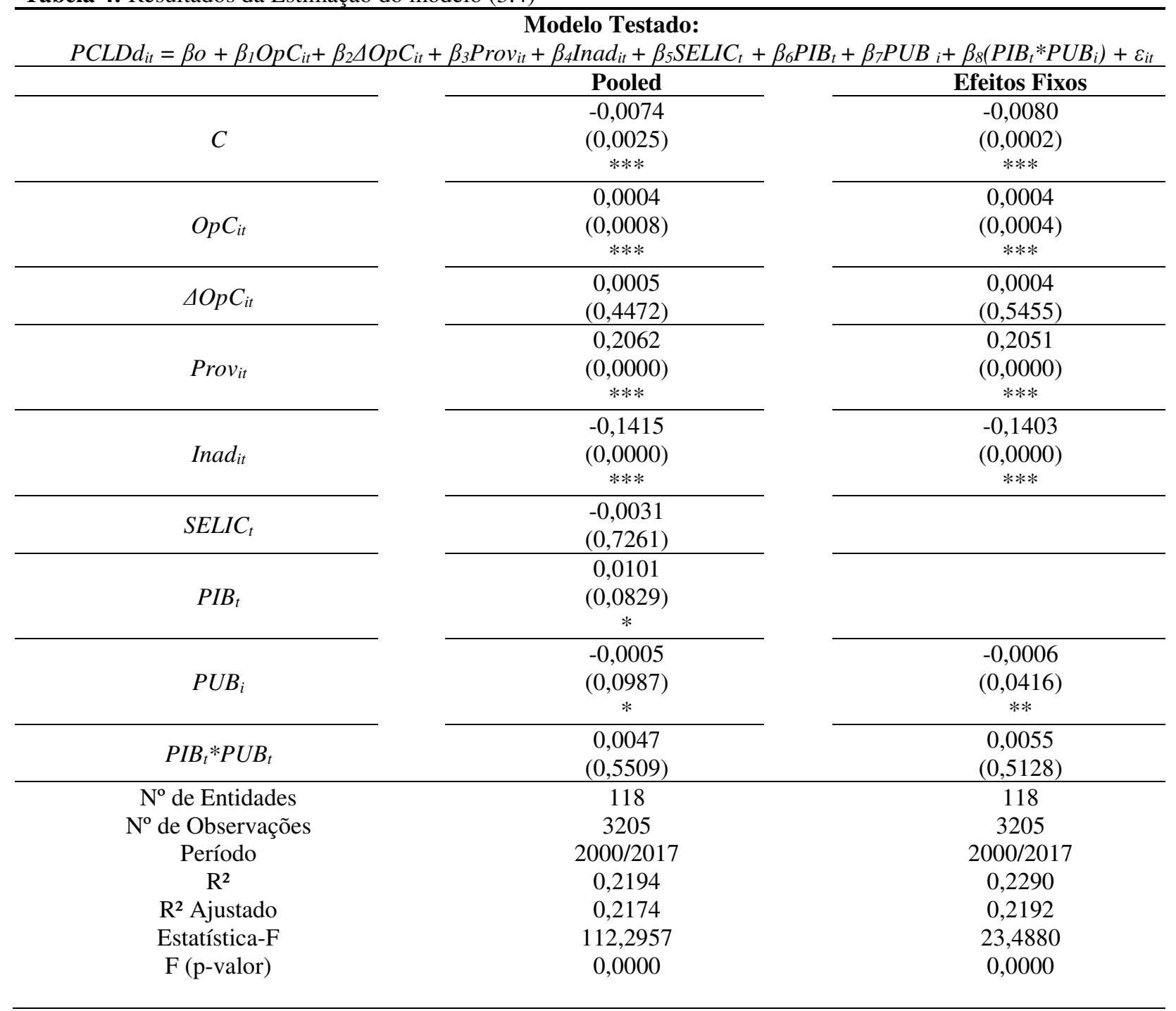


Onde: $\boldsymbol{P C L D d i t ~ r e p r e s e n t a ~ o ~ n i ́ v e l ~ d a ~ p r o v i s a ̃ o ~ d e ~ c r e ́ d i t o ~ p a r a ~ l i q u i d a c ̧ a ̃ o ~ d u v i d o s a ~ d i s c r i c i o n a ́ r i a , ~ o b t i d a ~ p o r ~ m e i o ~}$ da razão entre a PCLD discricionária e o total das operações de crédito do período; $\boldsymbol{O} \boldsymbol{p} \boldsymbol{C}_{i t}$ é o logaritmo natural do total das operações de crédito; $\boldsymbol{\Delta O} \boldsymbol{P} \boldsymbol{C}_{i t}$ é a variação proporcional do estoque das operações de crédito; Provit corresponde à razão entre a PCLD do período e o total das operações de crédito; Inad $_{i t}$ mede a razão entre o total das operações classificadas entre os níveis de risco "E" e "H" e o total das operações de crédito; $\boldsymbol{S} \boldsymbol{E} \boldsymbol{L} \boldsymbol{I} \boldsymbol{C}_{\boldsymbol{t}}$ é a taxa básica de juros da economia acumulada do semestre t; $\boldsymbol{P I} \boldsymbol{B}_{t}$ traduz a variação do PIB no semestre t; $\boldsymbol{P} \boldsymbol{U} \boldsymbol{B}$ é uma variável dummy, assumindo 0 para bancos privados e 1 para bancos públicos. Nível de significância: ***1\%, $* * 5 \%, * 10 \%$. P-valores entre parênteses.

Apesar de a relação entre variável de interesse $(\boldsymbol{P I B} * \boldsymbol{P U} \boldsymbol{B})$ e a variável dependente $(\boldsymbol{P C L D d})$ apresentar sinal positivo, não foi constatada relevância estatística, não corroborando com $\boldsymbol{H}_{2}$. Esse resultado indica que não é possível afirmar que os bancos públicos brasileiros possuem comportamento anticíclico, comparativamente aos bancos privados, no que diz respeito à constituição da PCLD discricionária. Nesse contexto, destaca-se que, apesar de a relação entre a variável de controle $(\boldsymbol{P U B})$ e a variável dependente $(\boldsymbol{P C L D d})$ possuir sinal negativo e apresentar relevância estatística, o que indica que os bancos públicos, em média, provisionam menos PCLD discricionária do que os bancos privados, não é possível afirmar que isso possui relação com os ciclos econômicos. Também aqui cabe a ressalva de que os testes empíricos se restringem ao exame do impacto do nível de atividade econômica na dimensão das provisões discricionárias para risco de crédito por parte dos bancos públicos em termos contemporâneos.

Em relação às variáveis de controle, foi constatada relação positiva e estatisticamente relevante entre as variáveis $\boldsymbol{P C L D d}$ e $\boldsymbol{O p C}$, o que indica que, quanto maior for a carteira de crédito, maior será a PCLD discricionária da instituição e vice-versa. Verificou-se também que não há associação relevante entre a variável independente $(\boldsymbol{\Lambda O} \boldsymbol{p C})$ e a variável dependente $\boldsymbol{P C L D d}$, e, desse modo, não é possível afirmar que há relação entre a variação do total da carteira de crédito e a PCLD discricionária. Ainda em relação à carteira de crédito, a variável independente que indica o quanto a PCLD representa do total da carteira de crédito (Prov) possui associação positiva e estatisticamente relevante com a variável dependente, revelando que, quanto maior for o nível de provisão, maior será o valor da PCLD discricionária e viceversa.

A variável que traduz a inadimplência (Inad), mensurada pela proporção da carteira classificada entre os níveis de risco "E" e "H" se revelou negativamente relacionada com a provisão discricionária $(\boldsymbol{P C L D d})$. Isso pode ser explicado pelo fato de que a Resolução CMN $\mathrm{n}^{\text {o }}$ 2.682/99 impõe maior nível de provisão obrigatória para os maiores níveis de risco, o que reduziria a necessidade de provisão adicional, aqui tratada como PCLD discricionária. Ou seja, 
como a regra regulamentar já impõe um alto nível de provisionamento, reduz a necessidade de provisão discricionária complementar.

Em relação às variáveis macroeconômicas, os resultados revelam, inicialmente, não haver associação entre a variável dependente e a taxa básica de juros (SELIC). Já em relação ao nível de atividade econômica, os testes revelam associação positiva entre as variáveis $\boldsymbol{P I B}$ e a variável dependente PCLDd. Esse resultado pode ser entendido como um indicativo do comportamento anticíclico dos bancos brasileiros, em que ocorre o afirmado por Bouvatier e Lepetit (2012) sobre o fato de o efeito dos ciclos econômicos sobre as provisões ser compensado pela formação de um colchão para cobrir perdas com empréstimos.

\section{CONCLUSÃO}

Este estudo tem por objetivo identificar se os bancos públicos, comparativamente aos bancos privados, atuam de maneira anticíclica no que diz respeito ao mercado de crédito, seja em relação ao volume de crédito ou à política de reconhecimento da provisão para perdas associadas ao risco de crédito, tendo por base a estimação de modelos com o uso de dados em painel e dados semestrais de 2000 a 2017 de 118 bancos em atuação no mercado brasileiro. A expectativa do comportamento anticíclica se fundamenta na premissa documentada pela literatura de que os bancos públicos buscam objetivos além do retorno dos recursos investidos, buscando ampliar as oportunidades de desenvolvimento econômico, podendo exercer papel anticíclico e estabilizador em momentos de dificuldades macroeconômicas.

No que se refere à concessão de crédito, os testes empíricos demonstraram que a variação da carteira é positivamente relacionada com o nível de atividade econômica. Isso revela um comportamento pró-cíclico em relação à concessão de crédito por parte do conjunto dos bancos brasileiros, ou seja, aumentam de forma mais relevante a contratação de novas operações em momentos de crescimento econômico e reduzem esse movimento em momentos de retração. Ao se isolar as informações dos bancos públicos, o sinal encontrado é negativo coerente com a premissa do comportamento anticíclico - mas a relação encontrada não é estatisticamente relevante, resultado na não corroboração da hipótese de pesquisa. A combinação das duas relações revela que, não obstante a não confirmação da hipótese que previa a atuação anticíclica dos bancos públicos, esses registram comportamento distinto dos bancos privados. Isso porque no conjunto da amostra verifica-se um claro comportamento prócíclico dos bancos brasileiros no mercado de crédito, mas ao se isolar os bancos públicos a relação se inverte, embora sem relevância estatística. 
Quanto ao reconhecimento da provisão para perdas, os resultados empíricos evidenciam que o conjunto dos bancos brasileiros apresentam comportamento anticíclico, reconhecendo mais PCLD discricionário em períodos de maior crescimento econômico, o que permite criar um colchão para se prevenir em momentos de retração econômica - movimento compatível com a prática de gerenciamento de resultados. Em relação aos bancos públicos, não foi identificado comportamento distinto dos bancos privados, não corroborando a hipótese de pesquisa que previa essa distinção.

Como principal limitação da pesquisa há que se ressaltar o fato de que o método empregado para os testes empíricos não considera o peso de cada instituição analisada no mercado de crédito. Assim, os resultados refletem o comportamento dos bancos brasileiros públicos e privados - de forma geral, mas não leva em consideração a participação de cada um deles no mercado de crédito. Dada a característica de forte concentração do mercado bancário brasileiro, talvez essa seja uma variável relevante a se considerar. Outro aspecto a se considerar em futuros estudos é a análise do comportamento dos bancos públicos, segregando-os em controle da União e dos Estados, dada a possibilidade de apresentarem políticas e potencialidades distintas.

\section{REFERÊNCIAS}

ARAÚJO, A. M. H. B. A ciclicidade da provisão para créditos de liquidação duvidosa em bancos comerciais. Tese (doutorado em Ciências Contábeis) - Programa Multi-Institucional e Inter-Regional de Pós-Graduação em Ciências Contábeis UnB/UFPB/UFRN, 2014.

BASEL COMMITTEE ON BANKING SUPERVISION (BCBS). Principles for the management of credit risk. Basel: BIS, 2000. Disponível em: <www.bis.org>. Acesso em: 30 abr. 2018.

BEATTY, A.; LIAO, S. Do delays in expected loss recognition affect banks' willingness to lend? Journal of Accounting and Economics, v. 52, n. 1, p. 1-20, 2011.

BEBCZUK, R.; ET AL. A new look into credit procyclicality: International panel evidence. BCRA Working Paper Series 201155, Central Bank of Argentina, Economic Research Department. 2011.

BIKKER, J. A.; HU, H. Cyclical patterns in profits, provisioning and lending of banks and procyclicality of the new Basel capital requirements. Banca Nazionale del Lavaro Quaterly Review, v. 55, p. 143-175, 2002.

BIKKER, J. A.; METZEMAKERS, P. A. J. Bank provisioning behaviour and procyclicality. Journal of International Financial Markets, Institutions \& Money, v. 15, n. 2, p. 141-157, 2005 . 
BOUVATIER, V.; LEPETIT, L. Banks' procyclical behavior: Does provisioning matter? Journal of International Financial Markets, Institutions \& Money, v.18, n.5, p.513-526, 2008.

BOUVATIER, V.; LEPETIT, L. Provisioning rules and bank lending: a theoretical model. Journal of Financial Stability, v. 8, n. 1, p. 25-31, jan. 2012.

BUSHMAN, R. M.; WILLIAMS, C. D. Accounting discretion, loan loss provisioning, and discipline of Banks'risk-taking. Journal of Accounting and Economics, v. 54, n.1, p. 1-18, 2012.

CANECA, R. L; LUSTOSA, P. R. B. Ciclos econômicos e provisão para créditos de liquidação duvidosa nos bancos brasileiros. In. USP International Conference in Accounting, 17. 2017, São Paulo. Anais... São Paulo: USP, 2017.

CHAVES, V. F. A Importância da atividade financeira na economia e o papel do Estado na regulação do mercado de capitais. In. Encontro Nacional do CONPEDI, 23. 2014, Florianópolis. Anais... Florianópolis: UFSC, 2014.

SEABRA, F.; GIGLIO, R. F.; FERNANDES, R. L.; MEURER, R. Existe comportamento diferenciado entre bancos públicos e privados em relação à política monetária? Pesquisa \& Debate, v.20, n.1, p.97-111, 2009.

JAYME JR., F. G.; CROCCO, M. (Orgs.). Bancos públicos e Desenvolvimento. Rio de Janeiro: IPEA, 2010.

LAEVEN, L.; MAJNONI, G. Loan loss provisioning and economic slowdowns: too much, too late? Journal of Financial Intermediation, v. 12, n. 2, p. 178-197, apr. 2003.

LIS, S. F.; PAGÉS, J. M.; SAURINA, J. Credit growth, problem loans and credit risk provision in Spain. Bank of Spain, Working Paper 0018, 2000.

LONGBRAKE, W. A.; ROSSI, C. V. Procyclical versus countercyclical policy effects on financial markets. The Financial Services Roundtable, July, 2011.

MARTINS, T. S.; BORTOLUZZO, A. B.; LAZZARINI, S. G. Competição bancária: comparação dos comportamentos de bancos públicos e privados. Revista de Administração Contemporânea, v. 18, n. Ed.Esp., p. 86-108, 2014.

MATTHEY, A. Do public banks have a competitive advantage? European Journal of Finance, v. 16, n. 1/2, p. 45-55, 2010.

MENDONÇA, H.F.; DEZORDI, L. L.; CURADO, M. L. A determinação da taxa de juros em uma economia sob metas para inflação: o caso brasileiro. Indicadores Econômicos FEE, v. 33, n. 3, p. 97-110, dez. 2005

MICCO, A.; PANIZZA, U. Bank ownership and lending behavior. Econonmic Letters, v. 93, n. 2, p. 248-254, 2006. 
PRIMO, U. R. Relação da Provisão para Crédito de Liquidação Duvidosa das Instituições Financeiras do Brasil com a Inadimplência do Setor. In. Congresso USP de Controladoria e Contabilidade, 3. 2003, São Paulo. Anais... São Paulo: USP, 2003.

OLIVEIRA, G. C. D. O comportamento recente do crédito e da estrutura patrimonial de grandes bancos no Brasil (2002-2005): uma abordagem pós-keynesiana. In. Encontro da Sociedade Brasileira de Economia Política, 11. 2006, Vitória. Anais... Niterói: SEP, 2006.

STOLF, W.A. Quantificação do risco de crédito: um estudo de caso utilizando o modelo Creditrisk. Dissertação (Mestrado em Ciências). Universidade de São Paulo (USP), Piracicaba, 2008.

VASCONCELOS, M. R.; FUCIDJI, J. R.; SCORZAFAVE, L. G.; ASSIS, D. L.. O todo e as partes: uma análise da desigualdade de crédito entre os estados brasileiros e os determinantes do crédito bancário com a aplicação de dados em painel. Economia e Sociedade, v. 13, n. 1, p. 123-149, 2004.

YEYATI, E.; MICCO, A.; PANIZZA, U. A reappraisal of state-owned banks. Economía, v. 7, n. 2, p. 209-247, 2007. 